\title{
Patients - voulez-vous contribuer à la formation?
}

\section{Valérie Junod}

Prof. Dr, professeure aux Universités de Genève et Lausanne

La Cour européenne des droits de l'homme a pour rôle de défendre les droits de l'homme contre les atteintes - grandes ou petites - que leur portent les Etats membres. Une de ces atteintes a fait l'objet d'un arrêt récent sur le droit à l'autodétermination des patients [1]. Sans doute parce que la Suisse n'était pas mise en cause, l'arrêt est passé largement inaperçu [2]. Toutefois, l'affaire est transposable à la Suisse et pourrait avoir des conséquences sur la communauté médicale.

De quoi s'agit-il? Une femme avait accouché dans un hôpital public russe. Son accouchement (par voie basse) avait été pénible et compliqué, mais l'enfant était finalement né en bonne santé. La plainte de la mère portait sur la (simple) présence de médecins étudiants pendant l'accouchement [3]. Lors de son admission à l'hôpital, la patiente avait été prévenue par brochure qu'une telle présence était

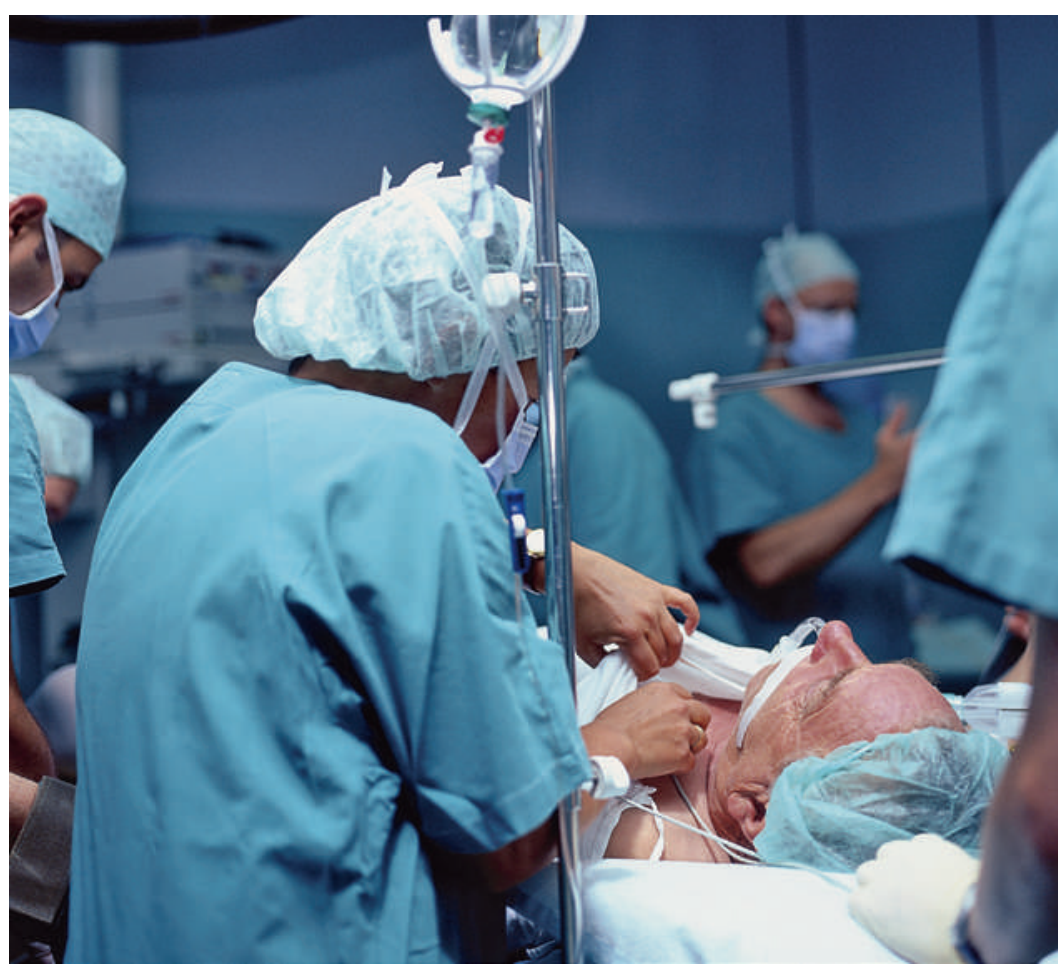

Sans consentement libre et éclairé du patient, les étudiants ne devraient pas participer à son traitement. possible [4]. Elle avait objecté, mais sans succès, l'hôpital n'acceptant pas de telles objections, vu son statut (accessoire) d'institution de formation pour la relève médicale.

Qu'a jugé la Cour? Elle estime que la législation russe ne contenait pas la base légale précise qui aurait été nécessaire pour imposer aux patients la présence comme observateurs de médecins étudiants. Il existait certes une disposition légale dans la loi sur la santé [5]; cependant, celle-ci était trop générale et annonçait de surcroît une législation de mise en œuvre qui n’avait pas été adoptée. La Cour a également pris

La plainte de la mère portait sur la présence de médecins étudiants pendant l'accouchement.

en considération l'état de vulnérabilité de la femme qui accouche: la patiente était fatiguée, avait été placée en sommeil thérapeutique à deux reprises et n'était plus (au moment des faits) en mesure de choisir un autre hôpital. Ayant conclu à l'absence de base légale suffisante, la Cour s'est dispensé de décrire les mesures de précaution à mettre en place pour que la participation de médecins étudiants soit jugée conforme au droit du patient au respect de sa sphère privée. Si la Cour de Strasbourg a évidemment tenu compte de la nature de l'intervention médicale ici en cause (un accouchement difficile) [6], cette circonstance n'est pas signalée comme décisive dans l'arrêt, de sorte que la portée de celui-ci ne se limite nullement à la gynécologie / l'obstétrique.

Que faut-il en retenir pour la Suisse? Comme ailleurs dans le monde, la pratique qui consiste à faire participer les médecins étudiants aux soins est très répandue en Suisse, en particulier dans les hôpitaux universitaires [7]. Les étudiants assistent passivement au début de leur formation, puis progressivement participent à l'administration des traitements. Les patients n'en sont pas toujours informés de manière formelle - c'est-à-dire précise, voire écrite. Certains hôpitaux annoncent la couleur dans des brochures remises à l'admission du patient (voir le tableau infra décrivant les pratiques des cantons romands). 
D'autres se contentent d'une information orale lors du passage de l'équipe médicale. Les patients ne savent pas toujours s'ils peuvent objecter à une telle présence et quelle conséquence aura leur objection. L'hôpital est un endroit déroutant, voire déstabilisant, pour le patient qui vient y chercher soins et assistance. La plupart des patients se sentent en position d'infériorité par rapport aux soignants - et ils le

\section{Le patient devrait être renseigné.}

sont en réalité. Si certains cantons ont légiféré sur ce thème (tabl. 1), le droit fédéral suisse ne contient pas de disposition légale permettant, et a fortiori, imposant la présence de médecins observateurs.

Faut-il pour autant changer les pratiques en Suisse? A l'évidence, un médecin ne peut se former correctement sans être au contact des patients. Il est tout aussi évident qu'un étudiant doit commencer par observer, avant de prendre part aux soins, puis finalement d'en assumer la responsabilité. Il est difficile, voire socialement impensable, d'envisager un autre système de formation; ainsi, on n'imaginerait guère de cantonner la formation dans des institutions spécialisées qui ne soigneraient que des patients volontaires.

Si le principe d'une participation des étudiants aux soins auprès des patients ne doit en aucun cas être changé, les modalités de cette participation doivent être rendues plus explicites et plus transparentes.
Dans l'idéal, pour pouvoir se prononcer valablement, le patient devrait être renseigné sur:

- qui sont les observateurs (par ex. leur sexe et leur nombre),

- quel est leur niveau de formation (par ex. débutant ou avancé),

- quelle est leur formation (par ex. médecin ou infirmière),

- quel va être leur rôle (par ex. purement passif ou contribuant au diagnostic),

- quelle va être la durée de leur présence (par ex. pour un soin isolé ou tout au long du séjour),

- quelle sera l'intimité aménagée pendant celle-ci (par ex. dans une salle individuelle ou auprès du lit du patient aux côtés d'autres malades),

- quel va être l'encadrement du médecin diplômé (par ex. toujours présent ou se fiant aux rapports des étudiants),

- quel sera le droit d'accès des étudiants au dossier médical du patient,

- quel sera le «retour» (feed-back) fait au patient sur son implication dans la démarche de formation (par ex. l'étudiant a correctement effectué la procédure médicale),

- quels sont ses droits (par ex. possibilité de révoquer en tout son consentement).

Aujourd'hui, une discussion approfondie sur les points cités ci-dessus est clairement l'exception, plutôt que la règle. Au demeurant, le peu de temps à disposition

Tableau 1: Gros plan sur les hôpitaux universitaires romands et leurs lois cantonales.

Le thème de la participation des patients à la formation est abordé dans la majorité des lois cantonales. II l'est également dans des brochures (au demeurant parfois longues) et/ou sur les sites web de la plupart des hôpitaux universitaires. Les formulations diffèrent toutefois entre les cantons; dans un même canton, le contenu de la brochure diverge parfois du texte de loi.

Canton Existe-t-il une disposition spécifique dans L'hôpital public fournit-il au patient une explication écrite (hôpital public) la loi cantonale? soit via internet, soit par une brochure qui lui est remise?

Genève Consentement du patient requis (art. 65); Passage dans un guide du patient hospitalisé (52 pages), dis(HUG) aucune précision sur les modalités ponible sur internet et remis à l'entrée.

Le passage en cause est descriptif (sur la possible présence d'étudiants), sans mention du droit du patient de consentir ou de refuser.

Fribourg Consentement du patient requis (art. 70e); Bref passage dans l'un des 5 guides pratiques (18 pages) dis(HFR) aucune précision sur les modalités ponible sur internet et aussi sur une page web sur les droits et responsabilités du patient.

Le passage en cause est général, sans mention du droit du patient de consentir ou de refuser.

\begin{tabular}{lll}
\hline $\begin{array}{l}\text { Neuchâtel } \\
\text { (HNE) }\end{array}$ & Non & Non \\
\hline Valais & $\begin{array}{l}\text { Consentement du patient requis (art. 43); } \\
\text { aucune précision sur les modalités }\end{array}$ & Non \\
\hline $\begin{array}{l}\text { Vaud } \\
\text { (CHUV) }\end{array}$ & $\begin{array}{l}\text { Droit de refuser du patient seulement si la } \\
\text { collaboration à l'enseignement l'expose "à } \\
\text { des désagréments importants»; le patient } \\
\text { doit être informé de ce droit (art. 25d) }\end{array}$ & $\begin{array}{l}\text { Pages) disponible sur internet et remise à l'entrée. } \\
\text { et brochure explique les raisons d'être de cette collaboration } \\
\end{array}$ \\
& & et reprend le langage de la loi s'agissant du droit de refuser. \\
\hline
\end{tabular}


du personnel hospitalier rend difficile, sinon impossible, un réel dialogue sur l'ensemble de ces points. Quel compromis faut-il alors envisager pour l'avenir? Sans être idéale, la solution concrètement concevable consisterait à informer plus systématiquement le patient de ses droits dès l'entrée à l'hôpital. Un patient qui accomplit les formalités administratives auprès du personnel d'accueil est mieux en mesure de recevoir une explication et de comprendre les raisons justifiant la présence d'étudiants. S'il a des questions, il sera plus enclin à les poser au secrétariat administratif; un dialogue peut se nouer où le patient exprime ses craintes et le personnel administratif les reçoit.

\section{Dans la plupart des cas, le patient saisira la nécessité de la formation médicale.}

Dans la plupart des cas, le patient saisira la nécessité de la formation médicale et donc la nécessité de la présence d'étudiants. Plusieurs études ont d'ailleurs montré que les patients à qui on offre le choix sont dans leur majorité ravis de contribuer à la formation [8]. Dans de rares cas, le patient s'y opposera, notamment pour des raisons sociales ou culturelles. La qualité de la formation reçue par le personnel hospitalier (secrétariat à l'accueil et équipes médicales) est également décisive pour faciliter la discussion avec le patient.

Sans remettre en cause la vocation éducative et formative des hôpitaux, l'arrêt de la Cour européenne des droits de l'homme rappelle que le droit au respect de la sphère privée prime en principe sur l'objectif bien sûr légitime et de santé publique - de former adéquatement les médecins. Finalement cet arrêt met en avant l'indispensable entente entre soignants et patients. Sans consentement libre et éclairé du patient, la relation de confiance entre celui-ci et son médecin est ébranlée. Or la médecine repose - heureusement aujourd'hui encore - sur cette confiance.

\section{Remerciements}

L'auteure remercie C.-A. et B. Junod pour leurs commentaires critiques, ainsi que les services juridiques et médicaux des hôpitaux publics romands qui l'ont aimablement renseignée.

\section{Références}

1 Arrêt dans l'affaire Konovalova c. Russie du 9 octobre 2014 Sur le thème connexe du respect de la sphère privée des femmes accouchantes, voir aussi les récents (11 décembre 2014) arrêts de la même Cour dans les affaires Hanzelkovi c. République Tchèque, respectivement Dubská et Krejzová c. République tchèque.

2 Voir toutefois European Court of Human Rights finds medical students' observation violated patient's right to privacy in Konovalova v. Russia, site web de l'International Justice Resource Center, October 21, 2014

3 Les médecins étudiants n'avaient - semble-t-il - pas dispensé de soins, mais, tout en restant passifs, ils avaient eu accès à des informations sur la santé de la patiente.

4 Le passage était bref et disait seulement: «Nous vous demandons de respecter le fait que les soins médicaux dans notre hôpital sont combinés avec l'enseignement aux étudiants en obstétrique et gynécologie. Pour cette raison, tous les patients sont impliqués dans le processus de formation» (traduction libre).

5 Selon la disposition russe en cause, les étudiants sont autorisés à assister aux soins médicaux conformément aux exigences de leur programme et sous la supervision du personnel médical responsable de leurs études.

6 Pour retenir une ingérence dans le droit à la vie privée de la recourante, la Cour met en avant (\$41) d'une part, la nature sensible de la procédure médicale en cause, et d'autre part l'accès qu'ont eu les étudiants aux informations médicales sur la santé de la patiente.

7 Pratiquement tous les pays font participer (passivement ou activement) leurs médecins en formation à la dispensation des soins. A l'étranger, depuis plusieurs années déjà, des auteurs se sont penchés sur cette problématique. Voir par ex. Norma O'Flynn et al. Consent and confidentiality in teaching in general practice: survey of patients' views on presence of students. 315 BMJ p. 1142 (1997); Amanda Howe \& Janie Anderson, Involving patients in medical education, 327 BMJ p. 326 (2003); Reshma Jagsi \& Lisa Soleymani Lehmann, The ethics of medical education, 329 British

8 Cf. par ex. Katie Coleman \& Elizabeth Murray, Patients' views and feelings on the community-based teaching of undergraduate medical students: a qualitative study. Family Practice. 2002;19(2):183. 